\section{ERGODICITY OF NON-HOMOGENEOUS MARKOV CHAINS WITH TWO STATES}

$\mathrm{BX}$

I. KOZNIEWST A (WARSAW)

1. Introduction. The scope of this note is to compare different notions of ergodicity for non-homogeneous Markov chains with two states.

A non-homogeneous Markov chain of $r$ states, say $E_{1}, E_{2}, \ldots, E_{r}$, is defined, as it is known, [5], by the initial distribution of the system $D_{0}\left(p_{0 \mid 1}, p_{0 \mid 2}, \ldots, p_{0 \mid r}\right)$ and the transition matrices of probabilities $P_{n}=\left\{p_{a b}^{(n)}\right\}$ for $n=1,2, \ldots$ where $p_{0 \mid a}$ denotes the (absolute) probability that the system is at the initial moment in state $E_{a}$ and $P_{a b}^{(n)}$ the (conditional) probability that the system is in state $E_{b}$ at moment $n$ if it was in state $E_{a}$ at moment $n-1$.

Thus the distribution of the system at moment $n$ is $D_{n}=D_{0} \prod_{i=1}^{n} P_{1}$, and the evident relations

$\sum_{b=1}^{r} p_{a b}^{(n)}=1 \quad$ for $\quad n=1,2, \ldots \quad$ and $\quad \sum_{a=1}^{r} p_{n \mid a}=1 \quad$ for $\quad n=0,1, \ldots$

hold, and $D_{n}=\left(p_{n \mid 1}, p_{n \mid 2}, \ldots, p_{n \mid r}\right)$.

If we take into consideration the matrices

(1) $\quad H_{m n}=\prod_{i=m+1}^{n} P_{i}$ for $m=0,1, \ldots, n=1,2, \ldots$ and $m \leqslant n-1$,

their elements $h_{a b}(m, n)$ will denote the probability that the system is at moment $n$ in state $E_{b}$ if it was at moment $m$ in state $H_{a}$. The relation

$$
\sum_{b=1}^{r} h_{a b}(m, n)=1
$$

and the Chapman-Kolmogorov equation
(2)$$
\boldsymbol{H}_{m n}=\boldsymbol{H}_{m t} \boldsymbol{H}_{t n} \quad \text { for } \quad m<t<n
$$

will hold.

For non-homogeneous chains three important notions of ergodicity are known.

Kolmogorov's principle of ergodicity [3] takes for non-homogeneous chains the following form [6]:

$$
\lim _{n \rightarrow \infty}\left[h_{a b}(m, n)-h_{c b}(m, n)\right]=0 \quad \text { for } \quad m=0,1, \ldots
$$

According to Hajnal [2] a non-homogeneous chain is ergodic in the weak sense if

$$
\lim _{n \rightarrow \infty}\left[h_{a b}(0, n)-h_{c b}(0, n)\right]=0 .
$$

A chain is ergodic in the strong sense if

$$
\lim _{n \rightarrow \infty} h_{a b}(0, n)=h_{b} .
$$

This property of chains was already known by Markov [4].

Morover, one might define, for symmetry, the notion of non-homogeneous chain ergodic in the strongest sense as follows

$$
\text { (6) } \quad \lim _{n \rightarrow \infty} h_{a b}(m, n)=h_{b} \quad \text { for } \quad m=0,1,2, \ldots
$$

It is obvious from the above definitions that the ergodicity in the weak sense (4) is implied by each other ergodicity and that the ergodicity in the strongest sense implies as well the strong ergodicity as the ergodicity in Kolmorogov sense.

The following examples will illustrate the difference between the definitions.

\title{
EXAMPLES
}

1. If $P_{n}=\left(\begin{array}{cc}1 & 0 \\ 1 /(n+1) & n /(n+1)\end{array}\right), H_{k n}=\left(\begin{array}{cc}1 & 0 \\ 1-k /(n+1) & k /(n+1)\end{array}\right)$, then $\lim _{n \rightarrow \infty} H_{k n}$ $=\left(\begin{array}{ll}1 & 0 \\ 1 & 0\end{array}\right)$ for each $k=1,2, \ldots$, as $\lim _{n \rightarrow \infty}(k / n+1)=0$ for $k$ constant.

This is an example of a chain ergodic in all mentioned senses.

2. Let $P_{1}=\left(\begin{array}{ll}1 / 4 & 3 / 4 \\ 1 / 4 & 3 / 4\end{array}\right)$ and $P_{n}=\left(\begin{array}{cc}1-1 / n^{2} & 1 / n^{2} \\ 0 & 1\end{array}\right)$, for $n>1$.

Then $H_{0 n}=\left(\begin{array}{l}(n+1) / 8 n(7 n-1) / 8 n \\ (n+1) / 8 n(7 n-1) / 8 n\end{array}\right)$ for $n>1$ and the chain is ergodic in the weak and strong senses, without being ergodic in other senses.

3. If $P_{1}=\left(\begin{array}{ll}0,2 & 0,8 \\ 0,2 & 0,8\end{array}\right)$ and $P_{n}=\left(\begin{array}{ll}0 & 1 \\ 1 & 0\end{array}\right)$ for $n>1$, the chain is ergodic only in the weak sense. 
4. Let $P_{1}=\left(\begin{array}{cc}0,1 & 0,9 \\ 0,2 & 0,8\end{array}\right)$ and $P_{n}=\left(\begin{array}{ll}0 & 1 \\ 1 & 0\end{array}\right)$ for $n>1$.

This chain is not ergodic whichever would be the sense of the ergodicity treated.

5. If $P_{2 n-1}=\left(\begin{array}{ll}0,9 & 0,1 \\ 0,4 & 0,6\end{array}\right)$ and $P_{2 n}=\left(\begin{array}{cc}0,8 & 0,2 \\ 0,3 & 0,7\end{array}\right)$ for $n=1,2, \ldots$,

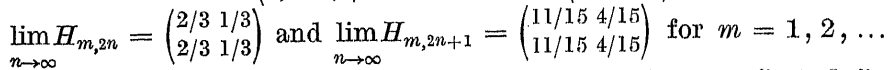

Thus the chain is ergodic in the sense of the two first definitions ((3) and (4)) and is not ergodic in other senses.

2. Necessary and sufficient conditions for ergodicity of two-state non-homogeneous Markov chains. For the Kolmogorov's definition of ergodicity we have

SIRASHDINOV THEOREM [6]. The necessary and sufficient condition for non-homogeneous two-state Markov chains to be ergodic in the sense of definition (3) is the divergence of the series

$$
\sum_{i=1}^{\infty}\left(1-\left|\lambda_{i}\right|\right)
$$

where $\lambda_{i}$ denotes the characteristic root, different from 1 , of matrix $P_{i}, i . e$. $\lambda_{i}=p_{11}^{(i)}-p_{21}^{(i)}$.

In the above theorem condition (7) is equivalent, as proved by Siragedino $\nabla$, to condition

$$
\prod_{i=m}^{\infty} \lambda_{i}=0 \quad \text { for } \quad m=1,2, \ldots
$$

Proof. For the sake of completeness we reproduce here Siragedinov's proof. We use the following property of the product of two stochastic matrices of the second order. The characteristic roots of such a product are products of "respective" roots of the factors. Hence if $P_{1}$ has 1 and $\lambda_{1}$ as roots, and $P_{2}$ has 1 and $\lambda_{2}$ as roots, $P_{1} P_{2}$ has 1 and $\lambda_{1} \lambda_{2}$ as roots. Applying it to (1) gives

$$
h_{11}(m, n)-h_{21}(m, n)=\prod_{i m m+1}^{n} \lambda_{i} \quad \text { for } \quad m=0,1, \ldots
$$

and

$$
\lim _{n \rightarrow \infty}\left[h_{11}(m, n)-h_{21}(m, n)\right]=\prod_{i=m}^{\infty} \lambda_{i},
$$

and this proves the theorem.
For a chain ergodic in the weak sense, as defined by Hajnal, we have

COROLLARY. Formula

$$
\prod_{i=1}^{\infty} \lambda_{i}=0
$$

gives a necessary and sufficient condition for the weak ergodicity (4) of non-homogeneous Markov two-state chains.

Now we can prove

THEOREM 1. The necessary and sufficient condition for the strongest ergodicity (6) of non-homogeneous Markov two-state chains is the divergence of

and the existence of

$$
\sum_{i=1}^{\infty}\left(1-\left|\lambda_{i}\right|\right)
$$

$$
\lim _{n \rightarrow \infty}\left(p_{21}^{(n)}+\sum_{i=2}^{n} p_{21}^{(i-1)} \prod_{k=i}^{n} \lambda_{k}\right) .
$$

Proof. Considering (2), the evident relation $P_{n}=H_{n-1, n}$ gives

(12) $\quad H_{m, n}=H_{m, n-1} P_{n} \quad$ valid for $\quad m=0,1, \ldots$ and $m<n$.

If we write this relation for elements instead for matrices we obtain

$$
h_{21}(m, n)=h_{21}(m, n-1) p_{11}^{(n)}+\left[1-h_{21}(m, n-1)\right] p_{21}^{(n)}
$$

$$
h_{21}(m, n)=h_{21}(m, n-1) \lambda_{n}+p_{21}^{(n)} .
$$

In particular,

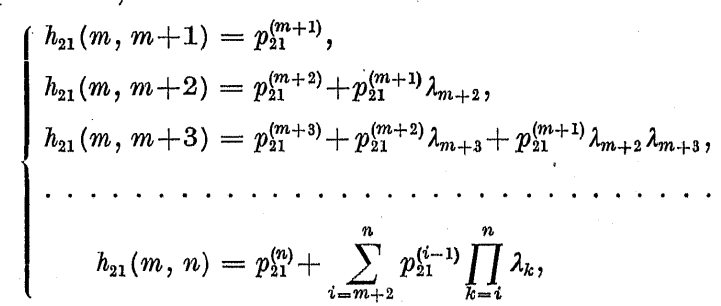

$h_{21}(m, n)$ will have a limit, for $m$ constant, and $n$ tending to infinity, if the right member of (14) has a limit. This limit will be the same as the limit of $h_{11}(m, n)$ if $\prod_{i=m}^{\infty} \lambda_{i}=0$. We can of course require the existence 
of

instead of that of

$$
\lim _{n \rightarrow \infty}\left(p_{21}^{(n)}+\sum_{i=2}^{n} p_{21}^{(i-1)} \prod_{k=i}^{n} \lambda_{k}\right)
$$

$$
\left(p_{21}^{(n)}+\sum_{i=m+2}^{n} p_{21}^{(i-1)} \prod_{k=i}^{n} \lambda_{k}\right) \quad \text { for each } m .
$$

CoRoLlaRY. $\prod_{i=1}^{\infty} \lambda_{i}=0$ and the existence of

$$
\lim _{n \rightarrow \infty}\left[p_{21}^{(n)}+\sum_{i=2}^{n} p_{21}^{(i \cdots 1)} \prod_{k=i}^{n} \lambda_{k l}\right]
$$

are necessary and sufficient conditions for the strong ergodicity (5) of nonhomogeneous Markov two-state chains.

In order to calculate

$$
\lim _{n \rightarrow \infty}\left[p_{21}^{(n)}+\sum_{i=2}^{n} p_{21}^{(i-1)} \prod_{k=i}^{n} \lambda_{k}\right]
$$

one has to apply Toeplitz theorem and this is generally cumbersome.

Anyhow, it is obvious that for chains ergodic in the weak sense if $\sum_{n=1}^{\infty} p_{21}^{(n)}$ is convergent, this limit exists.

Hajnal showed [2] that if $\prod_{i=1}^{\infty} \lambda_{i}=0$ and

(15) $p_{21}^{(n)}=\varrho p_{12}^{(n)}$ for $n=1,2, \ldots$ where $\varrho$ is a non-negative constant,

the chain is also ergodic in the strong sense.

Indeed, condition (15) entails then the existence of (11), as it is proved below. Then

Let us put $p_{21}^{(n)}=\varrho a_{n} /(\varrho+1)$ and $p_{12}^{(n)}=a_{n} /(\varrho+1)$ with $0 \leqslant a_{n} \leqslant 2$.

$$
\begin{aligned}
p_{21}^{(n)}+\sum_{i=2}^{n} p_{21}^{(i-1)} \prod_{k=2}^{n} \lambda_{k} & =\frac{\varrho}{\varrho+1}\left[a_{n}+\sum_{i=2}^{n} a_{i-1} \prod_{k=1}^{n}\left(1-a_{k}\right)\right] \\
& =\frac{\varrho}{\varrho+1}\left[a_{n}-\sum_{i=2}^{n}\left(1-a_{i-1}\right) \prod_{k=i}^{n}\left(1-a_{k}\right)+\sum_{i=2}^{n} \prod_{k=0=i}^{n}\left(1-a_{k}\right)\right] \\
& =\frac{\varrho}{\varrho+1}\left[a_{n}-\sum_{i=2}^{n} \prod_{k=i}^{n}\left(1-a_{k}\right)+\sum_{i=2}^{n} \prod_{k=1}^{n}\left(1-a_{k+1}\right)\right]
\end{aligned}
$$$$
\lambda_{n}=1-a_{n},
$$

$$
\begin{aligned}
& =\frac{\varrho}{\varrho+1}\left[a_{n}-\prod_{k=1}^{n}\left(1-a_{k}\right)+1-a_{n}\right] \\
& =\frac{\varrho}{\varrho+1}\left[1-\prod_{k=1}^{n}\left(1-a_{k}\right)\right]=\frac{\varrho}{\varrho+1}\left[1-\prod_{k=1}^{n} \lambda_{k}\right] .
\end{aligned}
$$

We see that if (9), then

$$
\lim _{n \rightarrow \infty}\left[p_{21}^{(n)}+\sum_{i=2}^{n} p_{21}^{(i-1)} \prod_{k=i}^{n} \lambda_{k}\right]=\frac{\varrho}{\varrho+1} .
$$

In order to compare conditions of ergodicity and to state several theorems, we define

Case (A). We are saying that case (A) of a chain is happening if in the sequence $\left\{P_{n}\right\}$ there exists such $k$ that $\lambda_{k}=0$ and that, for $n>k$, $\lambda_{n} \neq 0$ and $\prod_{i=k+1}^{\infty} \lambda_{i} \neq 0$.

If the case is considered from probabilistic point of view, $P_{k}$ with $\lambda_{k}=0$ indicates that at moment $k$ the transition probabilities are independent of issue states, and consequently the distribution of the system at moment $k$ is independent of the distribution at moment $k-1$, hence independent of the distribution at any previous time. Chains containing such $P_{k}$ may be considered rather as trivial cases of chains.

THEOREM 2. Ergodicities in Kolmogorov's sense (3) and in the weak sense (4) of non-homogeneous Markov two-state chains are equivalent if case (A) is excluded.

THEOREM 3. Ergodicities in the strong (5) and strongest (6) senses of non-homogeneous Markov two-states chains are equivalent if case (A) is excluded.

The proofs of these theorems follow from the comparison of conditions (8) and (9).

For case (A) we have

THEOREM 4. If there exists such $k$ that $\lambda_{k}=0$ and either

(a) $\quad \prod_{i=k+1}^{\infty} \lambda_{i}=c$ where $c \neq 0$,

$o r$

(b) $\prod_{i=k+1}^{\infty} \lambda_{i}$ is neither convergent nor divergent to 0 and

$$
\lim _{n \rightarrow \infty}\left[p_{21}^{(n)}+\sum_{i=2}^{n} p_{21}^{(i-1)} \prod_{k=i}^{n} \lambda_{k}\right]
$$


exists, the chain is ergodic in the strong sense without being ergodic in the strongest sense.

Proof. (a) As $\prod_{i=1}^{\infty} \lambda_{i}=0$, it remains to prove that

$$
\lim _{n \rightarrow \infty}\left[p_{21}^{(n)}+\sum_{i=2}^{n} p_{21}^{(i-1)} \prod_{k=i}^{n} \lambda_{k}\right]
$$

exists, in order to have the chain ergodic in the strong sense. The convergence of $\sum_{n=1}^{n} p_{21}^{(n)}$ would be sufficient for that purpose.

Indeed, the convergence of $\prod_{i=k+1}^{\infty} \lambda_{i}$ involves that of $\sum_{i=1}^{\infty}\left(1-\lambda_{i}\right)$ and that of $\sum_{i=1}^{\infty} p_{21}^{(i)}$ too, since $1-\lambda_{i}=p_{12}^{(i)}+p_{21}^{i=k+1}$. The chain cannot be ergodic in the strongest sense because $\prod_{i=n}^{\infty} \lambda_{i} \neq 0$ for $m>k$.

(b) is obvious.

The following two theorems are obvious:

THeorem 5. A non-homogeneous Markov two-state chain is ergodic in the Kolmogorov's sense (3) and is not ergodic in the strong sense (5) if

(a) there exists such $k$ that, for $n>k, \lambda_{n} \neq 0$ but $\prod_{i=1 / k+1}^{\infty} \lambda_{i}=0$ and there exists no

$$
\varliminf_{n \rightarrow \infty}\left[p_{21}^{(n)}+\sum_{i=2}^{n} p_{21}^{(i-1)} \prod_{k=i}^{n} \lambda_{k}\right],
$$

(b) there exists an infinity of $\lambda_{i}=0$ related to $P_{n_{1}}, P_{n_{2}}, \ldots$ but there exists no $\lim _{k \rightarrow \infty} P_{n_{k}}$.

THEOREM 6. If case (A) happens and

$$
\lim _{n \rightarrow \infty}\left[p_{21}^{(n)}+\sum_{i=2}^{n} p_{21}^{(i-1)} \prod_{k=1}^{n} \lambda_{k}\right]
$$

does not exist, the non-homogeneous Markov two-state chain is ergodic in the weak sense (4) and is not ergodio in any other sense.

3. Ergodicity of homogeneous Markov two-state chains. In the homogeneous case we have

THEOREM 7. For homogeneous Markov two-state chains the four treated ergodioities are equivalent.

Proof. For homogeneous chains $\lambda_{i}=\lambda$ is constant. Oase (A) cannot happen, thus on one hand the weak (4) and Kolmogorov's (3) ergodicities are equivalent and on the other hand the strong (5) and the strongest (6) ergodicities are equivalent too.
Hence it remains to prove the equivalence of weak and strong ergodicities. The condition for weak ergodicity, $\prod_{i=1}^{\infty} \lambda_{i}=0$, for homogeneous chain is $\lim _{i \rightarrow \infty} \lambda_{i}=0$ and that entails $|\lambda|<1$.

It follows from Fréchet [1] that homogeneous chains may be divided into four classes as regards the kind of characteristic roots of the transition matrix of probabilities:

1. $\lambda=1$ is a simple root and there is no other root of modul 1 .

This is the regular case of chains which are ergodic in the strong sense, i. e. $\lim p_{a b}^{(n)}=p_{b}$.

2. $\lambda=1$ is a multiple root and there is no other root of modul 1 .

These chains are not ergodic in the strong sense. Here $\lim p_{a b}^{(n)}=p_{a b}$.

3. $\lambda=1$ is a simple root and there exist other roots of modul 1 .

These chains are not ergodic in the strong sense, $\lim _{n \rightarrow \infty} p_{a b}^{(n)}$ does not exist, but

$$
\lim _{n \rightarrow \infty} \frac{1}{n} \sum_{i=1}^{n} p_{a b}^{(i)}=\pi_{b}
$$

(it is the so-called Cesarro ergodicity).

4. $\lambda=1$ is a multiple root and there exist other roots of modul 1 .

These chains are not ergodic in the strong sense, $\lim p_{a b}^{(n)}$ does not exist and

$$
\lim _{n \rightarrow \infty} \frac{1}{n} \sum_{i=1}^{n} p_{a b}^{(i)}=\pi_{a b} .
$$

Therefore $|\lambda|<1$ proves the strong ergodicity of the chain, since $\lambda$ is the second, besides 1 , root of the transition matrix.

\section{REFERENOES}

[1] M. Fréchet, Théorie des événements en ohaine dans le cas d'un nombro fini d'états, Recherches théoriques modernes sur le calcul des probabilités, 1952.

[2] J. Hajnal, The ergodio properties of non-homogeneous finite Markov ohains, Proc. Cambridge Phil. Soc. 52 (1) (1956), p. 67-77.

[3] A. N. Kolmogorov, Uber die analytisohen Methoden in der Wahrsoheinlichkeitsrechnung, Math. Ann. 104 (1931), p. 415-458.

[4] А. Марков, Исчисление веролтпостей, Москва 1913.

[5] В. И. Р ом ано в ск и й, Дискретнице чепи Маркова, Москва 1949.

[6] С. Н. Си р аж диинов, Эреодический принчип для неоднородных чепей маркова, Доклады АН СCCP 71 (1950), p. 829-834.

\footnotetext{
- Regu par la Rédaction le 25.1.1957
} 